\section{Amamentação e uso de antiinflamatórios não esteróides pela nutriz: informações científicas versus conteúdo em bulas de medicamentos comercializados no Brasil}

\author{
Breastfeeding and use of nonsteroidal \\ anti-inflammatory drugs by lactating \\ women: scientific information versus \\ patient information leaflets in medication \\ available in the Brazilian market
}

Roberto Gomes Chaves 1

Joel Alves Lamounier 2

Cibele Comini César 3

Mateus Alves Lima Corradi 4

Renata de Paula e Mello 5

Camila Martins Gontijo 6

Jaqueline Matos Drumond 7

\section{Abstract}

Objectives: confront information contained in non-steroidal anti-inflammatory drugs labels and information leaflets with the scientific data on their use during lactation.

Methods: a bibliographic review was performed using the terms: "breastfeeding", "lactating", "drugs", "medication", "non-steroidal anti-inflammatory drugs" and "information leaflets". The information obtained in articles and books were compared with the information in leaflets and labels on the use of non-steroidal anti-inflammatory drugs during the breastfeeding period.

Results: among the 27 non-steroidal anti-inflammatory drugs commercialized in Brazil, only 14 $(51.9 \%)$ contained safety information related to use during breastfeeding. In the information leaflets of ten non-steroidal anti-inflammatory drugs considered safe for use during breastfeeding, nine (90\%) had information in their leaflets to avoid use during breastfeeding, or to discontinue breastfeeding. In the information leaflets of 11 of the 13 non-steroidal, anti-inflammatory drugs (84.6\%) which had no information on the use by lactating women there was a suggestion to either avoid use or discontinue breastfeeding.

Conclusions: information contained in the leaflets is not consistent with the scientific evidence related to non-steroidal anti-inflammatory drugs use and breastfeeding. There's a need of further knowledge related to non-steroidal anti-inflammatory drugs and their use during breastfeeding.

Key words Breast feeding, Lactation, Pharmaceutical preparations, Medicine package inserts

\section{Resumo}

Objetivos: confrontar as informações contidas nas bulas de medicamentos antiinflamatórios não esteróides com as evidências científicas do uso desses fármacos durante a amamentação.

Métodos: foi realizada revisão bibliográfica nas bases de dados LILACS e MEDLINE, utilizando os termos: "amamentação", "lactação", "drogas", "medicamentos", "antiinflamatórios não esteróides" e "bulas". As informações obtidas em artigos e livros foram confrontadas com o conteúdo das bulas sobre o uso dos AINEs durante a lactação.

Resultados: dentre os 27 antinflamatórios não esteróides comercializados no Brasil foram encontradas referências sobre segurança para uso durante a amamentação em apenas 14 (51,9\%). Dos dez antinflamatórios não esteróides considerados como seguros para uso durante a lactação, nove (90\%) continham informação em bula para evitar uso nesse período ou suspender a amamentação. Na bula de 11 aos $13(84,6 \%)$ antinflamatórios não esteróides carentes de informações sobre uso pelas nutrizes foi sugerido evitar seu uso ou suspender a amamentação.

Conclusões: as informações contidas nas bulas são discordantes das evidências científicas a respeito da compatibilidade dos antinflamatórios não esteróides com a amamentação. Há necessidade de maior conhecimento acerca da segurança desses medicamentos durante a amamentação.

Palavras-chave Amamentação, Lactação, Preparações farmacêuticas, Bulas de medicamentos 


\section{Introdução}

O aleitamento materno está associado a benefícios de ordem nutricional, imunológica, cognitiva, afetiva, econômica e social. Portanto, urge conhecer os fatores que se relacionam ao desmame precoce, no intuito de permitir maior tempo de amamentação para as crianças. ${ }^{1} \mathrm{O}$ uso de medicamentos durante a amamentação é um tema de grande importância prática, visto a associação descrita entre essa prática e o desmame, além da frequiente necessidade do uso de medicamentos durante algum momento da lactação. ${ }^{2}$ Os antiinflamatórios não esteróides (AINEs) estão entre os fármacos mais utilizados em todo o mundo, inclusive durante a lactação. ${ }^{3-6}$ Vários estudos relatam ser a maioria dos AINEs seguros para uso durante a lactação.5,7-9 Estudo de revisão sobre a utilização de fármacos por nutrizes e os efeitos adversos em lactentes, mostrou que esses fármacos foram responsáveis por $8 \%$ das reações descritas. Somente em seis lactentes foram relatados efeitos desta natureza, sendo dois por ácido acetilsalicílico e um caso para dipirona, indometacina, naproxeno e paracetamol.10

Entretanto, informações em bulas sobre o uso desses fármacos durante a lactação são às vezes carentes e/ou mesmo contraditórias. Observa-se que as bulas não seguem um princípio básico que facilite a compreensão do conteúdo, o que na maior parte das vezes pode gerar confusão por parte do usuário. 11 Além disso, a indústria farmacêutica tende a uma postura na qual o uso de medicamentos durante a amamentação é baseado quase sempre em razões legais e não em razões clínicas. ${ }^{9}$ Esta posição contribui para que informações equivocadas ou ausência de informações corretas sobre o uso do fármaco durante a lactação possam constituir importante fator para interromper amamentação. Portanto, torna-se necessário melhor investigação sobre os efeitos desta categoria de fármacos, em especial dos novos AINEs, sobre os lactentes durante a amamentação. É importante, também que o conteúdo científico das bulas seja melhor elaborado, podendo servir como veículo de informação correta e segura, não somente para o profissional de saúde como para a população em geral.

A Agência Nacional de Vigilância Sanitária (ANVISA), órgão responsável pela regulamentação de informações em bulas de medicamentos, não incluiu na Resolução de Diretoria Colegiada (RDC) $\mathrm{n}^{\circ} 140$, de 29 de maio de 200312 exigências para inclusão de informações científicas sobre o uso de medicamentos durante a amamentação. Desse modo, permite aos laboratórios utilizar nas bulas infor- mações carentes de bases científicas que desestimulam a prática do aleitamento materno, privando o lactente dos inúmeros benefícios do leite humano. É importante ressaltar que a bula para o profissional de saúde é documento legal sanitário que contém informações técnico-científicas e orientações sobre medicamentos para o seu uso racional. Para o paciente, a bula contém informações sobre o medicamento, devendo ser escrita em linguagem apropriada, ou seja, de fácil compreensão. Informações tais como advertências e contra-indicações que facilitem o uso correto e seguro do medicamento fazem parte da bula, como forma de prevenir agravos à saúde. Porém, muitas vezes a bula pode não informar corretamente ou mesmo deixar uma lacuna de informações importantes em se tratando do uso de medicamentos durante a lactação.

A carência de estudos sobre os efeitos do uso de medicamentos pela nutriz sobre o lactente também é considerada fator associado ao desmame na vigência do uso de medicamentos pela nutriz. ${ }^{13} \mathrm{Na}$ última revisão da American Academy of Pediatrics (APP), 8 sobre uso de medicamentos durante a lactação são incluídos apenas 233 fármacos. A mais ampla e mais recente revisão acerca do tema foi realizada por Hale 9 sendo incluídos 774 fármacos. Se considerarmos que no Brasil são comercializados aproximadamente 1500 fármacos, 14 faltam informações sobre uso na lactação de 686 (45,7\% do total).

O objetivo desse estudo consiste em confrontar as informações contidas nas bulas de medicamentos antiinflamatórios não esteróides com as evidências científicas do uso desses fármacos durante a amamentação. A carência de trabalhos publicados sobre esse tema, reforça a importância de um estudo dessa natureza como contribuição para ajustes e modificações nas bulas.

\section{Métodos}

Foi realizada uma revisão bibliográfica utilizando os Descritores na Área de Ciências da Saúde (DeCS), e o seu correspondente em inglês Medical Subjects Headings (MeSH), vocabulários de acesso às bases de dados LILACS e MEDLINE, sendo os termos pesquisados em português "amamentação", "lactação", "drogas", "medicamentos", "antiinflamatórios não esteróides" e "bulas"; e os termos correspondentes em inglês: "breastfeeding", "lactation", "drugs", "medicines", "nonsteroidal antiinflammatory", "label" e "patient information leaflets" (PILs).

Foram encontradas apenas duas referências que avaliaram a influência das informações de bulas 
Fármacos antiinflamatórios não esteróides (AINEs) disponíveis no Brasil e respectivos nomes comerciais e laboratórios.

\begin{tabular}{|c|c|c|}
\hline Classes farmacológicas & Fármacos & Nomes comerciais (laboratórios) \\
\hline Salicilatos & Ácido acetil salicílico & Aspirina (Bayer) \\
\hline \multirow[t]{3}{*}{ Derivados pirazolônicos } & Fenilbutazona & Butazona (Boehringer) \\
\hline & Oxifembutazona & Tandrex* (Sintofarma) \\
\hline & Dipirona & Novalgina (Aventis Farma) \\
\hline \multirow[t]{2}{*}{ Derivado do ácido antranílico (fenamato) } & Ácido mefenâmico & Ponstam (Pzifer) \\
\hline & Ibuprofeno & Benotrin (EMS) \\
\hline \multirow[t]{3}{*}{ Derivados do ácido propiônico } & Naproxeno & Flanax (Roche) \\
\hline & Cetoprofeno & Profenid (Aventis Pharma) \\
\hline & Loxoprofeno & Loxonin (Sankyo) \\
\hline \multirow[t]{2}{*}{ Derivados do ácido indolacético } & Indometacina & Indocid (Prodrome) \\
\hline & Glucametacina & Teoremin (Asta Médica) \\
\hline \multirow[t]{2}{*}{ Derivados do ácido fenilacético } & Diclofenaco & Biofenac (Aché) \\
\hline & Aceclofenaco & Proflam (Bristol-Myers Squibb) \\
\hline \multirow[t]{2}{*}{ Derivado do ácido pirrolacético } & Cetorolaco & Deocil (Diffucap-Chemobras) \\
\hline & Piroxicam & Feldene (Pfzier) \\
\hline \multirow[t]{2}{*}{ Derivados do oxicam } & Tenoxicam & Tilatil (Roche) \\
\hline & Meloxicam & Movatec (Boehringer Ingelheim) \\
\hline Derivado do ácido carbâmico & Flupirtina & Katadolon (Asta Medica) \\
\hline Derivado da fenoximetanossulfanilida & Nimesulida & Nisulid (Asta Medica) \\
\hline Derivado indazólico & Benzidamina & Benflogin (Aché) \\
\hline \multirow[t]{2}{*}{ Derivado para-aminofenol } & Paracetamol & Tylenol (Janssen-Cilag) \\
\hline & Celecoxib & Celebra (Pharmacia) \\
\hline \multirow[t]{3}{*}{ Inibidores seletivos da COX-2 } & Etoricoxib & Arcoxia (Merk Sharp e Dohme) \\
\hline & Lumiracoxib & Prexige (Novartis) \\
\hline & Parecoxib & Bextra IM/IV (Pfizer) \\
\hline \multirow[t]{2}{*}{ Outros } & Mepirizol (Epirizol) & Mebron (Nikko) \\
\hline & Clonixinato de Lisina & Dolamin (Sintofarma) \\
\hline
\end{tabular}

* Associação oxifembutazona, hidróxido de alumínio e trissilicato de magnésio.

sobre lactação. 10,12 Portanto, foi necessário utilizar informações de livros e manuais técnicos a fim de fornecer subsídios para realização desse artigo.

A lista dos antiinflamatórios não esteróides atualmente disponíveis para comercialização no Brasil foi adquirida através da Gerência Geral de Medicamentos da ANVISA, sendo listados 27 fármacos (Tabela 1). As bulas dos medicamentos foram consultadas a partir das publicações do Dicionário de Especialidades Farmacêuticas (DEF) 14 e Phisician Reference (PR) Vade-Mécum, 15 do software Enciclopédia de Produtos Farmacêuticos (EPF) Millennium 16 e do Bulário eletrônico da ANVISA.17 Nessa categoria foi incluído também o paracetamol que, embora possua baixa atividade antiinflamatória, tem largo uso como analgésico em nosso meio. ${ }^{4}$ Não foram incluídos nesse estudo os AINEs comercializados em forma farmacêutica exclusiva para administração por via tópica devido ao baixíssimo risco de transferência para o leite materno.

O conteúdo das bulas foi, então, comparado com os estudos de revisão mais recentes e completos sobre o assunto: American Academy of Pediatrics, ${ }^{8}$ World Health Organization (WHO) ${ }^{18}$ Hale $^{9}$ e Hale et al. 19 Não foram encontrados estudos sobre a segurança para uso na lactação dos AINEs não citados nas referidas revisões. Para fim didático foi realizada uma adaptação da classificação de Hale 9 incluindo AINEs não citados nesta, porém constantes nas últimas revisões da American Academy of 
Tabela 2

Classificação dos antiiflamatórios não esteróides (AINEs) conforme nível de segurança para uso durante a lactação.

\begin{tabular}{ll}
\hline Classificação & Fármaco \\
\hline Seguros & $\begin{array}{l}\text { Ácido mefenâmico celecoxib, cetoprofeno, } \\
\text { cetorolaco, diclofenaco, dipirona, } \\
\text { fenilburazona, ibuprofeno, paracetamol, } \\
\text { piroxicam, }\end{array}$ \\
Moderadamente seguros & $\begin{array}{l}\text { Naproxeno (uso agudo), indometacina, ácido } \\
\text { acetil salicílico, meloxicam }\end{array}$ \\
Possivelmente perigosos & Naproxeno (uso crônico) \\
\hline
\end{tabular}

Fonte: Adaptado de Hale TW. Medications and mother's milk. Amarillo; 2004. 9

\section{Figura 1}

Informações contidas nas bulas sobre antiinflamatórios não esteróides (AINEs) seguros para uso durante a amamentação

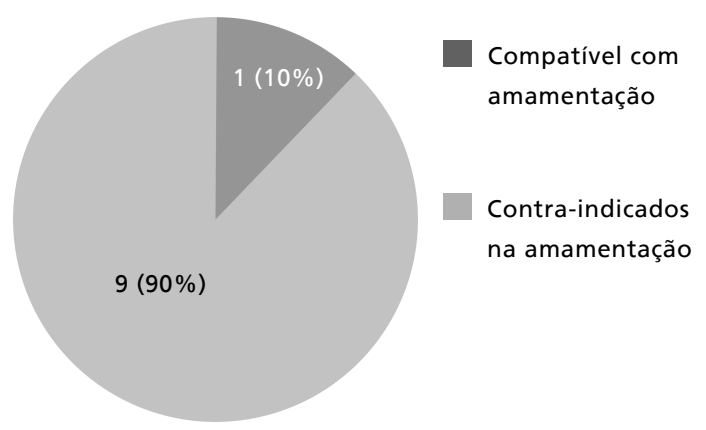

Figura 2

Informações contidas nas bulas sobre antiinflamatórios não esteróides (AINEs) considerados seguros e moderadamente seguros para uso durante a amamentação

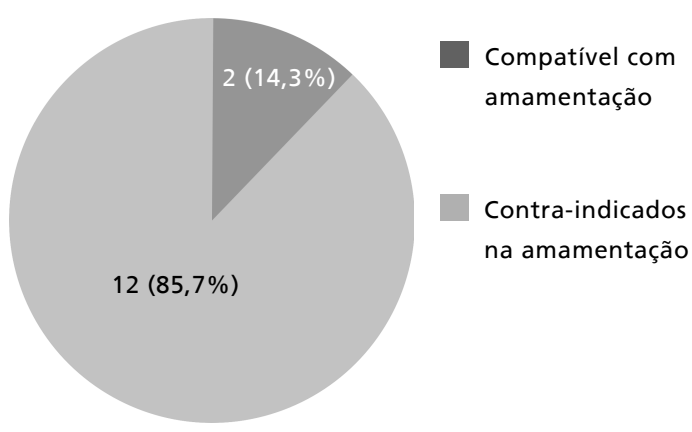

celecoxib, cetoprofeno, cetorolaco, diclofenaco, dipirona, fenilbutazona e piroxicam foram considerados de uso contra-indicados, sendo sugerido evitar o uso ou mesmo a suspensão da amamentação. A bula da dipirona orienta suspensão da amamentação até 48 horas após o seu uso.

A Figura 2 ilustra o conteúdo das bulas dos AINEs considerados seguros e moderamente seguros para uso na lactação. Como se pode observar, de 14 fármacos, 12 são considerados contra-indicados para uso durante esse período. 
Informações em bulas de medicamentos sobre o uso de antiinflamatórios não esteróides (AINEs) durante a lactação e seus efeitos adversos no lactente comparadas com dados publicados pela American Academy of Pediatrics (APP)(1) e por Hale.(2)

\begin{tabular}{|c|c|c|}
\hline Medicamento & Informações da bula & Informações científicas \\
\hline Aceclofenaco & Contra indicado na amamentação & Sem informação \\
\hline Acido acetilsalicílico & $\begin{array}{l}\text { Uso permitido. Não foram relatados efeitos } \\
\text { prejudiciais a bebês. }\end{array}$ & Moderadamente seguro(1,2) \\
\hline Ácido mefenâmico & Não deve ser utilizado durante amamentação. & Seguro(1,3) \\
\hline Benzidamina & Informar ao médico se está amamentando & Sem informação \\
\hline Celecoxib & Não utilizar durante a amamentação & Seguro(4) \\
\hline Cetoprofeno & Não recomendado & Seguro(2) \\
\hline Cetorolaco & Contra-indicado durante a lactação & Seguro(1,2) \\
\hline Clonixinato de lisina & Contra-indicado durante a lactação & Sem informações \\
\hline Diclofenaco & Não amamentar & Seguro(2) \\
\hline Dipirona & Evitar amamentar até 48 após uso do fármaco & Seguro(1) \\
\hline Etoricoxib & Descontinuar amamentação ou medicação & Sem informação \\
\hline Fenilbutazona & Suspender amamentação ou o tratamento & Seguro(1) \\
\hline Flupirtina & Não amamentar & Sem informação \\
\hline Glucametacina & Contra indicado durante amamentação & Sem informação \\
\hline Ibuprofeno & Não recomendado & Seguro $(1,2,3)$ \\
\hline Indometacina & Descontinuar medicamento ou droga & Moderadamente seguro $(2,3)$ \\
\hline Loxoprofeno & Contra indicado durante amamentação & Sem informação \\
\hline Meloxicam & Contra indicado na amamentação & Moderadamente seguro(2) \\
\hline Mepirizol & Usar sob orientação médica & Sem informação \\
\hline Lumaricoxib & Contra-indicado durante a amamentação & Sem informação \\
\hline Naproxeno & Não recomendado & $\begin{array}{l}\text { Moderadamente seguro } \\
\text { (uso agudo)(2) } \\
\text { Possivelmente perigoso } \\
\text { (uso crônico)(2) }\end{array}$ \\
\hline Nimesulida & Contra-indicado durante amamentação & Sem informação \\
\hline Oxifembutazona & Suspender amamentação ou o tratamento & Sem informação \\
\hline Paracetamol & Usar por períodos curtos durante a amamentação & Seguro $(1,2,3)$ \\
\hline Parecoxib & Suspender o aleitamento ou o tratamento & Sem informação \\
\hline Piroxicam & Não recomendado durante amamentação & Seguro(1,2) \\
\hline Tenoxicam & Suspender aleitamento materno ou o medicamento & Sem informação \\
\hline
\end{tabular}

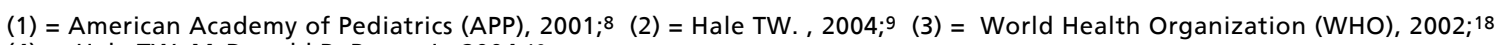

(4) = Hale TW, McDonald R, Boger J., 2004.19 
As bulas de treze AINEs não continham, estudos sobre sua segurança durante a lactação. Assim, sugere-se que seja evitado o uso durante a lactação ou suspensão da amamentação dos seguintes medicamentos: aceclofenaco, clonixinato de lisina, etoricoxib, flupirtina, glucametacina, loxoprofeno, lumaricoxib, nimesulida, oxifembutazona, parecoxib e tenoxicam. Dever-se-ia utilizar sob estrita orientação médica a benzidamida e o mepirizol. Portanto, na bula de onze dos treze $(84,6 \%)$ AINEs carentes de informações sobre uso pelas nutrizes sugere-se evitar seu uso ou suspender a amamentação.

A Tabela 3 mostra, resumidamente, a discrepância entre as informações contidas nas bulas dos AINEs com aquelas encontradas nas revisões da AAP, 8 WHO, 18 Hale 9 e Hale et al. 19

\section{Discussão}

Os AINEs estão entre os fármacos mais utilizados pela nutriz durante a lactação, principalmente no puerpério imediato. $20 \mathrm{Em}$ geral são prescritos, para o alívio de dor relacionada com intervenções cirúrgicas, cólicas uterinas e mastites. Bar-Oz et al.7 ressaltam que, devido ao grande número de fármacos analgésicos seguros para uso durante a lactação, não se deve permitir a sensação de dor pelas nutrizes nem a situação de se optar pelo fármaco ou pelo aleitamento. $\mathrm{Na}$ atualidade, os AINEs considerados mais seguros para uso durante a lactação são paracetamol e ibuprofeno 9,21 devido curto tempo de ação, ausência de metabólitos ativos e de relatos sobre efeitos adversos sobre o lactente.

Muitos estudos brasileiros demonstram a freqüente prática do uso de medicamentos durante a lactação. Um estudo com 2161 mulheres no pósparto imediato em maternidades de Belo Horizonte mostrou uso dos AINEs em 77,8\%.20 Em Itaúna, município próximo a capital mineira, pesquisa com 246 nutrizes revelou uso desse tipo de medicamentos pela quase totalidade $(99,6 \%)$ das puérperas no pósparto imediato, sendo prescritos 546 AINEs. Isso representa uma média de 2,2 fármacos deste grupo por puérpera. Os AINEs mais utilizados foram diclofenaco $(36,3 \%)$, clonixinato de lisina $(30,2 \%)$, cetoprofeno $(18,7 \%)$, dipirona $(13,5 \%)$, paracetamol $(0,7 \%)$, ácido acetilsalicílico $(0,4 \%)$ e tenoxicam $(0,2 \%)$. Após a alta hospitalar as nutrizes foram seguidas durante o período de 12 meses ou até a interrupção da amamentação. Também nesse período os AINEs foram a classe farmacológica mais utilizada, sendo a dipirona o fármaco mais usado. 6

A elevada freqüência da utilização dos AINEs pelas nutrizes reforça a necessidade de informações em bulas baseadas em dados científicos quanto ao uso desses fármacos durante a lactação. Porém, o presente estudo mostra que $90 \%$ dos AINEs sabidamente seguros para uso durante a lactação são considerados pela indústria farmacêutica como contra indicados durante esse período. Ou seja, existe uma contradição no conteúdo da bula com os dados e informações disponíveis em publicações científicas, gerando grande confusão tanto para o profissional de saúde quanto para a população em geral. A bula do ácido acetilsalicílico, considerado como "moderadamente seguro", devido a relatos de palidez, irritabilidade e acidose metabólica, ${ }^{22}$ menciona "não terem sido documentados problemas com seu uso durante a lactação". Apenas a bula do analgésico paracetamol (não usado como antiinflamatório) apresentou concordância com as evidências científicas. Tal achado confirma as afirmações de que as bulas de medicamentos sabidamente seguros durante a lactação contêm orientações que os contraindicam nesse período. 10 Pode-se, então, constatar uma falta de compromisso da grande maioria das empresas farmacêuticas com as evidências científicas do uso de AINEs e aleitamento materno. Essa situação, que deve ser vista com cuidado e de forma criteriosa pelos órgãos responsáveis pela regulamentação de comercialização de fármacos no que diz respeito às bulas. No intuito de orientar a indústria farmacêutica sobre a forma e conteúdo das bulas dos AINEs, Jones e Seager23 recomendam incluir nas mesmas apenas a informação "Informe ao médico se você está amamentando", evitando, assim, a divulgação de informações equivocadas.

Conforme mostrado nesse estudo quase metade $(48,1 \%)$ dos AINEs comercializados no Brasil carecem de informações científicas para uso durante a lactação. Nesse grupo observa-se que $84,6 \%$ possuem na bula recomendações para não usar o medicamento ou suspender amamentação. Do ponto de vista médico, a informação mais adequada a ser incluída na bula, deveria ser uma menção de avaliar o risco-benefício ou usar conforme recomendação médica. Portanto, muito além do que uma simples mensagem de não usar durante amamentação. $O$ elevado número de AINEs sem referência na literatura sobre sua segurança durante a lactação revela a necessidade de maior investimento em pesquisas sobre o tema.

Bjerrum e Foged 11 comprovaram informações confusas e divergentes nas bulas de diversos fármacos e o uso durante o aleitamento materno. Observaram que o mesmo medicamento continha informações diferentes nas bulas. Tal problema não 
ocorre somente no Brasil, Anderson et al.10 ressaltam o conflito entre as informações das bulas e as evidências científicas sobre o uso de medicamentos durante o aleitamento. Tais divergências levaram o Food and Drug Administration (FDA), órgão que regulamenta e autoriza a comercialização medicamentos, a propor alterações nas bulas no que se refere ao aleitamento materno.

As bulas deveriam, além de seguir normas, conter informações cientificamente comprovadas sobre a compatibilidade de cada fármaco com a amamentação. A ANVISA editou a Resolução RDC n0 140 em 29 de maio de 2003 "para atender as reivindicações de mudanças".12 Observa-se que houve preocupação com melhoria na linguagem, com uma bula específica para o paciente com palavras de fácil compreensão. Além disso, também valorização do respeito à prescrição médica e na inclusão de advertência para novos fármacos, durante cinco anos, que efeitos indesejáveis e não conhecidos podem ocorrer. Porém, apesar desses avanços, percebe-se a não obrigatoriedade, na referida resolução, da inclusão de informações científicas sobre o uso de medicamentos durante a amamentação, sendo exigido apenas a inclusão de "advertências e recomendações sobre o uso adequado de medicamentos por grupos de risco" ou "descrição das contra-indicações". 12 No entanto, isto pode resultar em interrupção da amamentação como decisão do profissional de saúde que muitas vezes não dispõem de informações seguras sobre o assunto. Na dúvida e na consulta de bulas, a alternativa poderá ser o desmame da criança em mães que necessitam fazer uso de medicamentos durante a lactação.

O difícil acesso aos serviços de saúde de qualidade para a maior parte da população brasileira induz o indivíduo a consumir medicamento na expectativa de adquirir saúde. Além disso, no país são encontramos vários medicamentos da classe AINEs vendidos legalmente sem receita médica,

\section{Referências}

1. Chaves RG, Lamounier JA. Uso de medicamentos durante a lactação. J Pediatr. [Rio J] 2004; 80 [Supl 5]: S189-S98.

2. Ito S. Drug therapy for breast-feeding women. N Eng J Med. 2000; 343: 118-26.

3. Frolich JC. The classification of NSAIDs according to the relative inhibition of cyclooxygenase isoenzymes. Trends Pharmacol Sci. 1997; 18: 30-4.

4. Rang HP, Dale MM, Ritter JM. Agentes antiinflamatórios e imunossupressores. In: Rang HP, Dale MM, Ritter JM Farmacologia. Rio de Janeiro: Guanabara Koogan; 2001. Cap. 13, p. 189-204. inclusive em balcões de supermercados, lanchonetes e bares. Essa realidade reforça a necessidade da inclusão de informações científicas nas bulas desses medicamentos, pois muitas vezes essa será a única informação que a nutriz poderá ter acesso sobre a segurança do medicamento durante a amamentação.

Assim, considerando-se o interesse da ANVISA sobre a necessidade de melhorar as informações nas bulas de medicamentos vendidos no país, apenas as de conteúdo científico, ainda será necessário constante fiscalização das mesmas, em especial o uso durante lactação. Um estudo para avaliar a estratégia de marketing utilizada pelas indústrias farmacêuticas, sob orientação de agências de publicidades e empresas de comunicação, na busca de elevar o consumo de medicamentos pela população, revelou infrações na resolução governamental sobre o assunto. Em 100 propagandas de medicamentos, tendo como base a Resolução de Diretoria Colegiada (RDC) $n^{\circ} 102$, publicada pela ANVISA em 30 de novembro de 2003, observou-se que todas comentem infrações em um ou mais artigos da resolução. 24

As bulas de medicamentos constituem muitas vezes a única fonte de informação que pacientes e profissionais de saúde têm sobre o uso dos fármacos durante a lactação. Conforme revelou esse estudo, as informações contidas nas bulas estão muito discordantes das evidências científicas a respeito da compatibilidade dos AINEs com a amamentação. Como conseqüência, nutrizes podem, desnecessariamente, deixar de usar o AINEs, agravando sintomas ou doenças, ou mesmo podem promover o desmame, privando o lactente dos inúmeros benefícios do aleitamento materno. Assim, a padronização pela ANVISA do conteúdo científico das bulas poderia facilitar o entendimento tanto por parte do profissional de saúde quanto do usuário. Outras categorias de medicamentos não incluídos nesse estudo podem também apresentar situação semelhante aos dos antiinflamatórios do conteúdo de bulas e o uso durante a amamentação.
5. Hale TW. Medications in breastfeeding mothers of preterm infants. Pediatr Ann. 2003; 32: 337-47.

6. Chaves RG. Situação do aleitamento materno e do uso de medicamentos pela nutriz no primeiro ano de vida da criança em Itaúna, MG [dissertação mestrado]. Belo Horizonte: Faculdade de Medicina da Universidade Federal de Minas Gerais; 2004.

7. Bar-Oz B, Bulkowstein M, Benyamini L, Greenberg R, Soriano I, Zimmerman D, Bortnik O, Berkovitch M. Use of antibiotic and analgesic drugs during lactation. Drug Saf. 2003; 26: 925-35. 
8. AAP (American Academy of Pediatrics). Committee on Drugs. The transfer of drugs and other chemicals into human milk. Pediatrics. 2001; 108: 776-89.

9. Hale TW. Medications and mothers'milk. 11. ed. Amarillo: Pharmasoft; 2004.

10. Anderson PO, Pochop LS, Manoguerra AS. Adverse drug reactions in breastfed infants: less than imagined. Clin Pediatr. 2003; 42: 325-40.

11. Bjerrum L, Foged A. Patient information leaflets-helpful guidance or a source of confusion? Pharmacoepidemiol Drug Saf. 2003; 12: 55-9.

12. ANVISA (Agência Nacional de Vigilância Sanitária). Resolução - RDC no 140, de 29 de maio de 2003. Disponível em: www.anvisa.gov.br/legis/resol/2003/rdc/ 140_03rdc.htm [2005 abr 9].

13. Ministério da Saúde. Secretaria de Políticas de Saúde. Área técnica de Saúde da Criança. Amamentação e uso de drogas. Brasília (DF); 2000.

14. DEF (Dicionário de Especialidades Farmacêuticas): 2004/2005. 34. ed. Rio de Janeiro: Publicações Científicas; 2006.

15. P.R. Vade-Mécum: 2005/2006. 10. ed. São Paulo: Soriak; 2005.

16. Enciclopédia de Produtos Farmacêuticos (EPF) Millennium. [programa para computador] São Paulo: Centralx, 2005.

17. ANVISA (Agência Nacional de Vigilância Sanitária). Bulário eletrônico. Disponível em http://bulario.bvs.br/ index.php . [2006 jan 15].

Recebido em 13 de junho de 2004

Versão final apresentada em 30 de maio de 2006

Aprovado em 14 de junho de 2006
18. WHO (World Health Organization), UNICEF (United Nations International Children's Emergency Fund) Breastfeeding and maternal medication: recommendations for drugs in the eleventh WHO model list of essential drugs. Geneva; 2002. Avalilable from: http://www.who.int/childadolescenthealth/NewPublications/NUTRITION/BF Maternal.edication.pdf. [2005 April 25].

19. Hale TW, McDonald R, Boger J. Transfer of celecoxib into human milk. J Hum Lact. 2004; 20: 397-403.

20. Lamounier JA, Cabral CM, Oliveira BC, Oliveira AB, Júnior AMO, Silva APA. O uso de medicamentos em puérperas interfere nas recomendações ao aleitamento materno? J Pediatr. [Rio J] 2002; 78: 57-61.

21. Spigset O, Hagg S. Analgesics and breast-feeding: safety considerations. Paediatr Drugs. 2000; 2: 223-38.

22. Clark JH, Wilson WG. A 16-day-old breast-fed infant with metabolic acidosis caused by salicylate. Clin Pediatr. 1981; 20: 53-4.

23. Jones R, Seager J. The ideal nonsteroidal anti-inflammatory drug patient information leaflet. Am J Med. 2001; 110: 38S$41 \mathrm{~S}$.

24. César NA. A persistirem os sintomas o médico deverá ser consultado: isto é regulação [dissertação mestrado]. Rio de Janeiro: Instituto de Medicina Social da Universidade do Estado do Rio de Janeiro; 2003. 